\title{
CONTROL DE MÚltiple OBJETIVO DEL MOTOR DE INDUCCIÓN EMPLEANDO ASIGNACIÓN DE ESTRUCTURAS PROPIAS Y ALGORITMOS GENÉTICOS
}

\author{
SANTIAGo SÁnCheZ ${ }^{1}$ \\ Jorge A. Zambrano ${ }^{2}$ \\ EdUARDo Giraldo ${ }^{3}$
}

\section{Resumen}

Este trabajo se centra en el diseño de un controlador lineal mediante la estrategia de optimización de múltiple objetivo sobre una máquina de inducción modelada en el sistema de coordenadas de flujo del rotor. Los índices de desempeño seleccionados son la sensibilidad de las variables de estado de forma individual, la ganancia del controlador y la sensibilidad mixta. La técnica de control empleada es la asignación de estructuras propias; la cual posiciona los polos usando los valores y vectores propios; la optimización es realizada con el método heurístico de algoritmo genético. Se realizaron los diseños del controlador de estructuras propias más control robusto con optimización de múltiple objetivo y un solo objetivo. Los resultados de simulación y comparación muestran que la optimización de múltiple objetivo lleva a la mejora del desempeño del sistema frente a la optimización de un solo objetivo y el control robusto $H_{\infty}$.

1 Centro de investigación, Instituto Tecnológico Metropolitano, santiagosanchez@ itm.edu.co

2 Grupo control automático, Universidad Tecnológica de Pereira, jzambrano@ohm. utp.edu.co

3 Programa de Ingeniería Eléctrica, Universidad Tecnológica de Pereira, egiraldos@ utp.edu.co

Fecha de recepción: 15 de marzo de 2010

Fecha de aceptación: 9 de junio de 2010 


\section{Palabras clave}

Algoritmos Genéticos, Control robusto, Asignación de estructuras propias, Sensibilidad, Motor de inducción.

\section{Abstract}

A linear controller is calculated by the use of a multiobjective optimization technique, and is applied over the induction machine; which is modeled in the oriented coordinate rotor flux system. The performance indexes chosen are sensitivity of state variables, closed loop gain and the mixed sensitivity. The method used is the eigenstructure assignment; in which, the objective is to determine the controller through pole placement using eigenvalues and eigenvectors; the optimization is realized by the genetic algorithms. The tests applied over the system imply the controller design in combination with the robust problem and genetic algorithms, and two cases are taken into account; one objective and multiobjective. The computational results and comparisons show that the multiobjective optimization increases the performance of the system against the one objective optimization and the $\mathrm{H} \infty$ controller.

\section{Keywords}

Eigenstructure assignment, Genetic Algorithms, Induction motor, Robust control, Sensitivity. 


\section{INTRODUCCIón}

El control de máquinas eléctricas rotativas se ha trabajado en diferentes campos, tanto los sistemas de control no lineal, como los controladores lineales; los modelos dinámicos estudiados son presentados en los diferentes marcos por Leonhard (2001), Bose (2002) y Chiasson (2005); donde este último describe el sistema de la máquina de inducción en el sistema de coordenadas de flujo del rotor, empleado en este trabajo como modelo para la prueba. La selección de la técnica de control varía en el grado de conocimiento del diseñador así como la complejidad de esta; esto puede llevar a obtener sistemas con elevado costo computacional y una posible variación de los parámetros empleados podría causar una inestabilidad (Chiasson, 1998; Mohanti \& De, 2006); algunas metodologías que reducen el costo de diseño y computacional del controlador sobre la máquina eléctrica son las técnicas lineales; algunas de estas emplean un regulador proporcional integral con sintonización por algoritmos genéticos de un modelo estimado con lógica borrosa (Ustun \& Dermitas, 2009).

El control descrito por Duval et al. (2006) con asignación de estructuras propias, hace uso del la optimización analítica que incrementa la complejidad de diseño y no garantiza una solución óptima global. El diseño del control de múltiple objetivo empleado en este trabajo consiste en definir claramente los índices de desempeño que debe presentar el controlador sobre el sistema y mejorarlos por medio de un método ya sea analítico o heurístico. Estos índices de desempeño consisten en la mejora de las propiedades robustas del sistema; entre estas propiedades se encuentra el garantizar al sistema una estabilidad nominal con sus siglas en inglés (NS), un comportamiento nominal (NP) y la estabilidad robusta (RS) (Whidborne et al., 2003) las anteriores propiedades se obtienen calculando la sensibilidad mixta.

Otros objetivos a considerar son minimizar la sensibilidad de los polos de forma individual y mejorar la ganancia del controlador, ambas clases de objetivos son incluidos en este trabajo por el 
hecho de determinar la respuesta del sistema en el tiempo bajo perturbaciones y de mejorar la ganancia de realimentación; ya que estos son elementos clave para las funciones de sensibilidad, sensibilidad complementaria, comportamiento nominal y estabilidad robusta. Para cumplir estas condiciones se utiliza la asignación de estructuras propias, esta técnica de diseño considera al mismo tiempo el cálculo de un controlador y la determinación de la sensibilidad en el dominio de la frecuencia; de forma que se crean unas funciones de desempeño y se usa un modelo heurístico para mejorar el comportamiento de estas funciones de acuerdo a las necesidades de diseño (Liu \& Patton, 1997; 1998a; Whidborne et al., 2003).

El modelo de optimización heurístico usado para este trabajo son los algoritmos genéticos (AG). Debido a su manera de plantear la búsqueda del valor óptimo; estos algoritmos son aplicados a una gran cantidad de problemas con tiempo de respuesta muy competitivo con otras técnicas de optimización. Este trabajo está organizado por tres partes, la siguiente sección da una explicación del modelo de la máquina de inducción, la asignación de estructuras propias, sintonización de múltiple objetivo y optimización del control de múltiple objetivo, la tercera sección presenta los resultados de simulación y la cuarta sección muestra las conclusiones obtenidas de la aplicación del controlador.

\section{Metodología}

\subsection{Modelo del motor de inducción}

El modelo del motor de inducción emplea el sistema de coordenadas de flujo orientado del rotor descrito en (Leonhard, 2001; Chiasson, 1998; 2005), lo cual simplifica el diseño del controlador. El sistema es representado mediante un sistema de espacio de estados a partir de (1) hasta (5). 


$$
\begin{gathered}
\frac{d \omega}{d t}=\mu \varphi_{d} i_{d}-\frac{T_{L}}{J} \\
\frac{d i_{d}}{d t}=\left(\frac{\eta L_{m}}{\sigma L_{s} L_{m}}\right) \phi_{d}-\gamma i_{d}+n_{p} \omega i_{q}+\eta L_{m} \frac{i_{q}^{2}}{\phi_{d}}+\frac{u_{d}}{\sigma L_{s}} \\
\frac{d i_{q}}{d t}=-\left(\frac{L_{m} n_{p}}{\sigma L_{s} L_{r}}\right) \omega \phi_{d}-\gamma i_{q}-n_{p} \omega i_{d}-\eta L_{m} \frac{i_{q} i_{d}}{\phi_{d}}+\frac{u_{q}}{\sigma L_{s}} \\
\frac{d \phi_{d}}{d t}=-\eta \phi_{d}+\eta L_{m} i_{d} \\
\frac{d \rho}{d t}=n_{p} \omega+\eta L_{m} \frac{i_{q}}{\phi_{d}}
\end{gathered}
$$

Donde $\theta$ es la posición angular [rad], $\omega$ la velocidad angular $[\mathrm{rad} / \mathrm{s}], i_{d}$ e $i_{q}$ representa las corrientes en el estator para el eje directo y el eje en cuadratura $[A], \phi_{d}$ es la magnitud del campo de flujo del rotor $[W b]$, y $\rho$ es el ángulo del campo de flujo del rotor $[\mathrm{rad}], u_{q}, u_{d}$ la entrada del sistema son los voltajes del estator [V]. Los parámetros del estator y del rotor son descritos por $L_{s}$ la inductancia en el estator, $L_{r}$ la inductancia en el rotor y $L_{m}$ la inductancia de acoplamiento [H], $R_{s}$ y $R_{r}$ las resistencias en el estator y el rotor $[\Omega], J$ el momento de inercia $\left[\mathrm{kgm}^{2}\right], f$ la constante de amortiguamiento viscoso $[\mathrm{Nms}], n_{p}$ corresponde a los pares de polos y $T_{L}$ es el toque de carga $[N m]$. Además, $\sigma=1-L_{m} /\left(L_{r} L_{s}\right)$ es el factor de acoplamiento, $\eta=R_{r} / L_{r}$ el inverso de la constante de tiempo y para simplificar se asumen y $\mu=n_{p} L_{m} /\left(J L_{r}\right)$.

Para obtener un modelo lineal del sistema se asume que el ángulo del flujo del rotor puede ser estimado mediante la observación de los estados que actúan en (5) y que las otras variables de estado no dependen de este, además se puede hacer lineal el conjunto de ecuaciones compuesto por (1) a (4) alrededor del punto de operación $\left(\omega_{0}, \phi_{0}, i_{d 0}, i_{q 0}, \rho_{0}, u_{d 0}, u_{q 0}\right)$ (Sánchez et al., 2008). Con las entradas $V_{d}=90 \mathrm{~V}, V_{q}=0.9 \mathrm{Vy} T_{L}=5 \mathrm{Nm}$ y un punto de equilibrio $x_{0}=\left[\phi_{d}\right.$; $\left.i_{q} ; i_{d} ; \omega ; T_{e}\right]=[0.16 ; 17.98 ; 39.87 ; 234.46 ; 5]$ el sistema lineal se puede expresar por las matrices de espacio de estado de (7) hasta (9). 


$$
\begin{gathered}
\mathrm{B}=\left[\begin{array}{cc}
0 & 0 \\
\frac{1}{\sigma L_{s}} & 0 \\
0 & \frac{1}{\sigma L_{s}} \\
0 & 0
\end{array}\right] \\
\mathbf{C}=\left[\begin{array}{cccc}
1 & 0 & 0 & 0
\end{array}\right] \\
\mathbf{A}=\left[\begin{array}{cccc}
-\frac{f}{J} & 0 & \mu \phi_{d 0} & \mu i_{q 0} \\
n_{p} i_{q 0} & -\gamma & n_{p} \omega_{0}+2 \eta L_{m} \frac{i_{q 0}}{\phi_{d 0}} & \frac{\eta L_{m}}{\sigma L_{s} L_{r}}-\frac{\eta L_{m} i_{q o}^{2}}{\phi_{d o}^{2}} \\
-\frac{L_{m} n_{p} \phi_{d o}}{\sigma L_{r} L_{s}}-n_{p} i_{d 0} & -n_{p} \omega_{o}-\frac{\eta L_{m} i_{q o}}{\phi_{d 0}} & -\gamma-\eta L_{m} \frac{i_{d 0}}{\phi_{d 0}} & -\frac{n_{p} L_{m}}{\sigma L_{r} L_{s}} \omega_{0}+\frac{\eta L_{m} i_{q o} i_{d o}}{\phi_{d o}^{2}} \\
0 & \eta L_{m} & 0 & -\eta
\end{array}\right]
\end{gathered}
$$

El modelo ahora tiene cuatro variables de estado $x=\left(\Delta \omega, \Delta \mathrm{i}_{d}\right.$, $\left.\Delta i_{q}, \Delta \phi_{d}\right)$, dos entradas $u=\left(\Delta \mathrm{u}_{d}, \Delta u_{q}\right)$ y una salida $y=\Delta \omega$. El sistema puede ser expresado con (10) y (11).

$$
\begin{aligned}
& \dot{x}=A x+B u \\
& y=C x
\end{aligned}
$$

Donde $x \in R^{n}$ es el vector de estados, $u \in R^{m}$ es el vector de entradas en lazo abierto o la señal de control en lazo cerrado, $y$ $\in R^{r}$ corresponde al vector de salida, y $\mathrm{A} \in R^{n \times n}, \mathrm{~B} \in R^{n \times m}$ y $\mathrm{C} \in R^{r \times n}$ son las matrices del sistema.

\subsection{Asignación de estructuras propias}

El método empleado para el diseño del controlador es la asignación de estructuras propias en el cual se usa la estructura propia completa (valores propios, vector propio derecho e izquierdo) para el lazo cerrado, adicionalmente para este trabajo se usa una variación del problema original; considerando la solución del problema de robustez. Para calcular los parámetros requeridos por el sistema se procede con las etapas descritas a continuación. La primera etapa consiste en el análisis de la máquina en lazo abierto; una 
vez analizada la respuesta en lazo abierto, se procede de acuerdo a los criterios de diseño como respuesta en frecuencia, seguido se realiza la selección de los polos del sistema (valores propios) en lazo cerrado; los cuales se obtienen de forma que el sistema se base en una respuesta de un modelo de referencia y los vectores propios derechos e izquierdos de lazo cerrado son calculados por medio de una proyección ortogonal de los vectores propios de lazo abierto. Como segunda etapa, se ajusta el controlador modificando los valores propios de acuerdo al análisis de robustez (Liu \& Patton, 1997; 1998b; Whidborne, 2003).

El desarrollo de esta técnica consiste esencialmente de tres etapas. En el paso inicial se debe seleccionar la posición de los polos de acuerdo al número de variables de estado. El siguiente es hallar los vectores propios determinados a partir del paso anterior conviniendo con algunas estrategias de diseño y finalmente se calcula una ley de control apropiada para la selección de la estructura propia.

Para describir esta técnica, considere un sistema de espacios de estados de múltiple entrada y múltiple salida (MIMO) invariante en el tiempo tal y como se muestra en la Fig. 1.

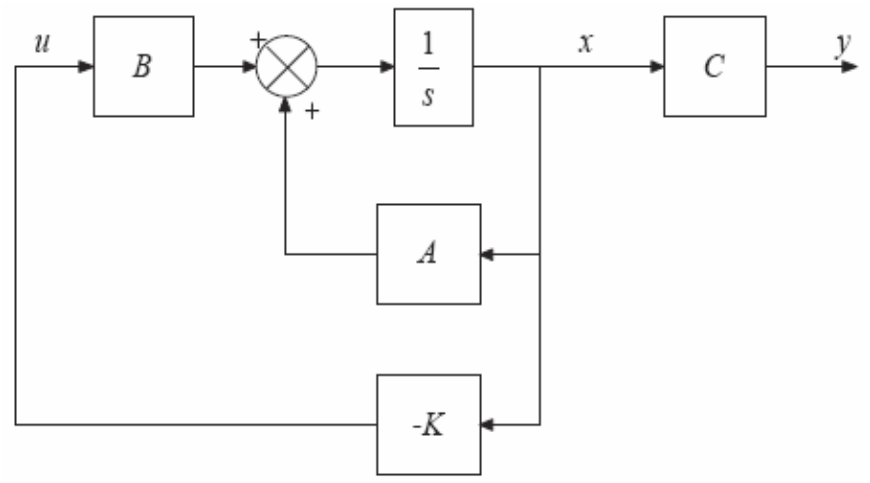

Fig. 1. Sistema de VARIAbles de Estados

Por medio de la Fig. 1, y empleando (10) y (11), se determinan dos fórmulas claves dentro del diseño. 
[150] Control de múltiple objetivo del motor de inducción empleando asignación de estructuras propias...

\subsubsection{Ley de control}

$$
u=K y
$$

Donde $\mathrm{K} \in R^{m \times p}$. Reemplazando (12) en (10) y a su vez reemplazándola en (11), se obtiene (13).

$$
x=(\mathrm{A}+\mathrm{BKC}) x
$$

De esa forma para la matriz (A+BKC), $\lambda_{\mathrm{i}}, v_{i} \in R^{n \times 1}, q_{i} \in R^{1 \times n}$ son los valores propios, los vectores propios derechos e izquierdos, respectivamente, donde $\boldsymbol{w}_{i} \in R^{m \times 1}$ queda definido en (14).

$$
w_{i}=K C v_{i}
$$

La siguiente proposición se emplea para la asignación de estructuras propias con realimentación fija (Liu \& Patton, 1997; Whidborne, 2002; 2003).

\subsubsection{Proposición}

Considere $\lambda_{\mathrm{i}}$ y $v_{\mathrm{i}}$ los cuales satisfacen el vector $w_{i}$

$$
\left[\begin{array}{ll}
\mathrm{A}-\lambda_{\mathrm{i}} I & \mathrm{~B}
\end{array}\right]\left[\begin{array}{c}
\mathrm{v}_{\mathrm{i}} \\
\mathrm{w}_{\mathrm{i}}
\end{array}\right]=0
$$

Con los valores de $v_{\mathrm{i}}, w_{\mathrm{i}}$ se crean las matrices $V=\left[v_{1} v_{2} \ldots v_{r}\right] y$ $W=\left[\begin{array}{llll}w_{1} & w_{2} & \ldots & w_{r}\end{array}\right]$; con ambos términos se determina el controlador $\mathrm{K}$ de la forma indicada en (16) (Duval et al., 2006).

$$
K=W(C V)^{-1}
$$

\subsection{Sintonización de múltiple objetivo}

Dentro de la técnica de diseño como es la asignación de estructuras propias, se obtienen un número determinado de funciones de desempeño que al ser optimizadas mediante un modelo analítico 
o numérico se genera un control de múltiple objetivo óptimo. Los objetivos usados en este controlador se describen a continuación.

\subsubsection{Sensibilidad del valor propio individual}

$$
\phi_{i}=\frac{\left\|\boldsymbol{q}_{i}\right\|\left\|_{2}\right\| \boldsymbol{v}_{i} \|_{2}}{\left|\boldsymbol{q}_{i}^{\mathrm{T}} \boldsymbol{v}_{i}\right|}
$$

Siendo $v_{i}$ y $q_{i}$ el i-ésimo vector propio derecho e izquierdo, para la matriz A+BKC. El principal objetivo es la minimización de las funciones de desempeño (Liu \& Patton, 1997; 1998a; 1998b; Whidborne et al., 2003).

\subsubsection{Control Robusto Ho}

El control robusto $\mathrm{H}_{\infty}$ corresponde a un función objetivo para determinar un controlador tal que el comportamiento nominal con sus siglas NP y la estabilidad robusta (RS) (ambos siendo propiedades robustas) sean garantizadas para un sistema de control. El problema $\mathrm{H}_{\infty}$ parte del problema de sensibilidad mixta descrita en (18) (Rodríguez \& López, 1996).

$$
\|\mathrm{H}\|_{\infty}=\phi_{\mathrm{n}+1}=\left\|w_{w_{T} T}^{w_{S}}\right\|_{\infty} \leq 1
$$

donde:

$$
\begin{aligned}
& S=\left(I-(S I-A)^{-1} B K\right)^{-1} \\
& T=K\left((S I-A)^{-1} B K-I\right)^{-1}
\end{aligned}
$$

Siendo $S$ la función de sensibilidad (19), $T$ la sensibilidad complementaria (20) y $W_{s}$ y $W_{T}$ las funciones de peso cuyo oficio es garantizar los objetivos de diseño mencionados con anterioridad y corresponden a un comportamiento de filtros pasa bajo o pasa alto de acuerdo al problema (Liu \& Patton, 1997; Whidborne et al., 2002; 2003). El objetivo para garantizar un buen desempeño con 
esta función se muestra en (21) y se define como la minimización sobre todo el conjunto de controladores $\mathrm{K}$.

$$
\min _{\mathrm{K}}\left\|\mathrm{w}_{\mathrm{S}} \mathrm{s}\right\|_{\infty}
$$

\subsubsection{Ganancia del controlador $K$}

Como otra función de desempeño, se consideran algunas restricciones en la ganancia de la matriz de control $\mathrm{K}$. Una medida de tipo escalar que refleja dicha restricción es (22).

$$
\phi_{\mathrm{n}+2}(\mathrm{~K})=\sqrt[2]{\left(\sum_{\mathrm{i}=1}^{\mathrm{m}} \sum_{\mathrm{j}=1}^{\mathrm{n}} \mathrm{K}_{\mathrm{ij}}^{2}\right)}
$$

donde $\mathrm{K}_{\mathrm{ij}}$ son los elementos de la matriz del controlador $\mathrm{K}$. Todas las funciones objetivo tienen como criterio de optimización las minimización de sus valores, por esta razón se formula la optimización dentro del problema de múltiple objetivo como se muestra en la expresión (23).

$$
\left\{\min _{\mathbf{K}, \boldsymbol{v}, u} \sum_{i=1}^{n+2} \frac{\phi_{i}(\boldsymbol{K}, \boldsymbol{v}, \boldsymbol{q})}{\varepsilon_{i}} ; \quad \phi_{i}(\boldsymbol{K}, \boldsymbol{v}, \boldsymbol{q})<\varepsilon_{i}\right.
$$

Donde $\varepsilon_{i}$ son valores positivos y se interpreta como los valores límites seleccionados por el diseñador para que estos índices de desempeño no superen los permitidos.

\subsection{Control de múlitiple objetivo óptimo}

En la etapa de sintonización del controlador se emplean las funciones descritas en (17), (18) y (22) y como modelo completo de optimización de múltiple objetivo (23), el método de optimización usado es el algoritmo genético que asume los pasos presentados en esta sub-sección (Whidborne, 2003). 
Paso 1. Representación del individuo

Cada solución en la población está representada como una cadena de números reales en lugar de una cadena binaria. Está formada por un vector aleatorio $\mathrm{Z} \in R^{1 \times n}$ y la matriz $\mathrm{W}=\left[\mathrm{w}_{1}, \mathrm{w}_{2}, \ldots, \mathrm{w}_{\mathrm{n}}\right]$ $\in R^{n \times n}$, entonces la representación del individuo está dada en (24).

$$
\mathrm{P}=\left[\mathrm{Z}, \mathrm{w}_{1}{ }^{T}, \ldots, \mathrm{w}_{n}{ }^{T}\right]
$$

Los parámetros de $\mathrm{Z}$ al ser reemplazado en (25) generan el vector de valores propios dentro de un rango establecido por el diseñador.

$$
\mathrm{P}=\left[\mathrm{Z}, \mathrm{w}_{1}^{\mathrm{T}}, \mathrm{w}_{2}^{\mathrm{T}}, \ldots, \mathrm{w}_{\mathrm{n}}^{\mathrm{T}}\right]
$$

donde $\lambda_{i}$ y $\bar{\lambda}_{i}$ son los límites inferiores y superiores para cada uno de los valores propios asignados.

Paso 2. Generación de la población inicial

Aleatoriamente se genera primero los $N$ conjuntos de individuos $\mathrm{P}$, donde $N$ es un número impar.

Paso 3. Evaluación de las funciones de desempeño

Evaluar las funciones de desempeño $\phi_{i}\left(\mathrm{P}_{j}\right)$ para todo $N$ conjuntos de parámetros $\mathrm{P}_{j}$. por medio de (26) y (27).

$$
\begin{aligned}
& \Delta_{\mathrm{j}}=\max _{i=1,2, \ldots n+2} \phi_{\mathrm{i}}\left(\mathrm{P}_{\mathrm{j}}\right) / \varepsilon_{\mathrm{i}} \\
& \Phi_{J}=\sum_{i=1}^{n * 2} \phi_{i}\left(\mathbf{P}_{j}\right)
\end{aligned}
$$

Paso 4. Selección

De acuerdo a la aptitud de las funciones de desempeño para cada conjunto de parámetros, remover los $(N-1) / 2$ miembros más débiles de la población y reordenar los conjuntos de parámetros. La aptitud de las funciones de desempeño se mide por (28). 
[154] Control de múltiple objetivo del motor de inducción empleando asignación de estructuras propias...

$$
F_{j}=\frac{1}{\Delta_{j}} \text { para } j=1,2, \ldots, N
$$

Paso 5. Cruce

El cruce se realiza mediante un promedio en la función de cruce para generar los $(N-1) / 2$ descendientes. Este operador de cruce toma dos parientes en el cual son seleccionados del paso anterior y de esa forma produce un descendiente $\left(\mathrm{P}_{C j}\right)$ que es el resultado del promedio de los dos parientes. La función promedio de cruce está definida como (29).

$$
P_{(C j)}=\frac{P_{G(j+1)}+P_{G}}{2} \text { para } j=1,2, \ldots, \frac{N-1}{2}
$$

\section{Paso 6. Mutación}

Un operador de mutación se considera como un número real de arrastre. La función que se va a optimizar se considera como un proceso continuo con colinas y valles. Si se encuentra una buena colina y además se desea realizar un salto alrededor de ellas para alcanzar la máxima colina global, este número real genera este efecto. Este efecto lo que hace es un barrido a lo largo del individuo arrastrándolo hacia arriba o hacia abajo con un pequeño valor aleatorio, por lo que se representa como una probabilidad que depende del parámetro del operador. La operación de mutación se presenta en (30).

$$
\mathrm{P}_{\mathrm{Mj}}=\mathrm{P}_{\mathrm{Gj}}+\mathrm{d}_{\mathrm{m}} \varepsilon_{\mathrm{j}} \text { para } \mathrm{j}=1,2, \ldots, \frac{\mathrm{N}-1}{2}
$$

donde $d_{m}$ es el máximo valor por alterar y $\varepsilon_{j} \in[-1,1]$ corresponde a una selección aleatoria de la dirección que toma la mutación.

\section{Paso 7. Elitismo}

La estrategia de elitismo consiste en copiar los mejores parámetros impidiendo que los mejores individuos pierdan los parámetros en la generación, esto se hace con el propósito de incrementar la 
velocidad de dominación dentro de una población para crear un súper individuo haciendo que mejore el desempeño del algoritmo genético. Los mejores conjuntos de parámetros $\mathrm{P}_{b}$ se satisfacen por medio de (31).

$$
\mathrm{E}_{\mathrm{b}}=\min _{l}\left\{\mathrm{E}_{l}: \mathrm{E}_{l} \leq \mathrm{E}_{\mathrm{m}}-\alpha\left(\Delta_{l}-\Delta_{\mathrm{m}}\right) \text { y } \Delta_{l}=\Delta_{\mathrm{m}}+\delta\right\}
$$

donde, $\Delta_{m}$ se calcula con (32)

$$
\Delta_{\mathrm{m}}=\underset{\mathrm{j}=1,2, \ldots \mathrm{n}+2}{\min }\left\{\Delta_{\mathrm{j}}\right\}
$$

$\mathrm{E}_{m}$ y $\mathrm{E}_{l}$ corresponde a $\Delta_{m}$ y $\Delta_{l}, \mathrm{a}>1$ y $\delta$ es un número positivo puesto por el diseñador.

Paso 8. Nuevos descendientes

Se adicionan los (N-1)/2 nuevos descendientes a la población los cuales son generados aleatoriamente. Actualmente, los nuevos descendientes son formados por la mutación de los mejores parámetros $\mathrm{P}_{b}$ con una probabilidad definida en (33).

$$
\mathrm{P}_{\mathrm{Nj}}=\mathrm{P}_{\mathrm{b}}+\mathrm{d}_{\mathrm{n}} \varepsilon_{\mathrm{j}} \text { para } \mathrm{j}=1,2, \ldots \frac{\mathrm{N}-1}{2}
$$

donde $d_{n}$ corresponde a la nueva mutación por alterar y $\varepsilon_{j} \in[-1,1]$ corresponde a una variable aleatoria. Además la nueva población estará formada por un conjunto de parámetros $\mathrm{P}_{\mathrm{Mj}}\left(\mathrm{j}=1,2, \ldots \frac{\mathrm{N}-1}{2}\right)$, $P_{\mathrm{Nj}}\left(\mathrm{j}=1,2, \ldots, \frac{\mathrm{N}-1}{2}\right)$ y $\mathrm{P}_{\mathrm{b}}$.

Paso 9. Restricción de parada

Repite el paso 3 hasta que pase un valor finito de iteraciones o logre la convergencia. La población es considerada a tener convergencia cuando se cumple (34).

$$
\Delta_{\mathrm{j}}-\Delta_{\mathrm{b}} \leq \varepsilon, \text { para } \mathrm{j}=1, \ldots, \mathrm{N}
$$

donde $\varepsilon$ es un número positivo empleado como criterio de parada. 


\section{Resultados}

Esta sección presenta los resultados más trascendentes y la comparación de la aplicación de la técnica de control robusto sobre el modelo lineal de la máquina de inducción, el controlador de estructuras propias considerando el problema de robustez y su sintonización óptima mediante el algoritmo genético con optimización de un objetivo y múltiple objetivo. De (Sánchez et al., 2008) los parámetros del motor de inducción son: $\mathrm{R}_{\mathrm{s}}=1,7 \Omega$, $\mathrm{R}_{\mathrm{r}}=3,9 \Omega, \mathrm{L}_{\mathrm{r}}=\mathrm{L}_{\mathrm{s}}=0,014 \mathrm{H}, \mathrm{L}_{\mathrm{m}}=0,0117 \mathrm{H}, \mathrm{n}_{\mathrm{p}}=2, \mathrm{~J}=0,0011 \mathrm{kgm}^{2}$, $\mathrm{f}=0,00014 \mathrm{Nm} / \mathrm{rad} / \mathrm{s}$. El par del motor es considerado constante con los siguientes valores iniciales $\mathrm{u}_{\mathrm{qo}}=141,8 \mathrm{~V}, \mathrm{u}_{\mathrm{do}}=1,42 \mathrm{~V}$, $\mathrm{i}_{\mathrm{do}}=16,434 A, \mathrm{i}_{\mathrm{qo}}=11,910 A, \phi_{\mathrm{do}}=0,192 \mathrm{~Wb}$ y $\omega_{\mathrm{o}}=163,2 \mathrm{rad} / \mathrm{s} \mathrm{y}$ las siguientes funciones de peso $\mathrm{W}_{\mathrm{s}} \mathrm{y} \mathrm{W}_{\mathrm{T}}$ que son el resultado del empleo de una etapa inicial de diseño de controladores $\mathrm{H} \infty$ con comportamiento de filtro pasa bajo y frecuencia de corte $100 \mathrm{~Hz}$.

$$
\begin{aligned}
& \mathrm{W}_{\mathrm{s}}=\frac{408.41}{(\mathrm{~s}+408.41)\left(2.533 * 10^{-6} \mathrm{~s}^{2}+2.33 * 10^{-3} \mathrm{~s}+1\right)} \\
& \mathrm{W}_{\mathrm{T}}=\frac{\mathrm{s}\left(\mathrm{s}^{2}+1316 \mathrm{~s}+7.66^{*} 10^{5}\right)}{(\mathrm{s}+408.41)\left(2.533 * 10^{-6} \mathrm{~s}^{2}+2.33^{*} 10^{-3} \mathrm{~s}+1\right.}
\end{aligned}
$$

Para el algoritmo genético de la sección 2.4 se emplea la restricción en los valores propios de la máquina $-4000 \leq \lambda_{1,2,3,4}$ $\leq-20$, la población es seleccionada con 40 individuos, $d_{m}=0,03$, $d_{n}=0,08, \alpha=1,1, \gamma=0.1$, el número de generaciones es 31 , $F \in\left[0,01-10^{5}\right], \phi_{\mathrm{i}} \leq 769$, para $\mathrm{i}=1,2,3,4, \phi_{5} \leq 1, \phi_{6} \leq 10000$. Al emplear los pasos del algoritmo genético mencionados en la sección 2.4, la evolución de cada función objetivo se muestra desde la Fig. 2 hasta la Fig. 7. 


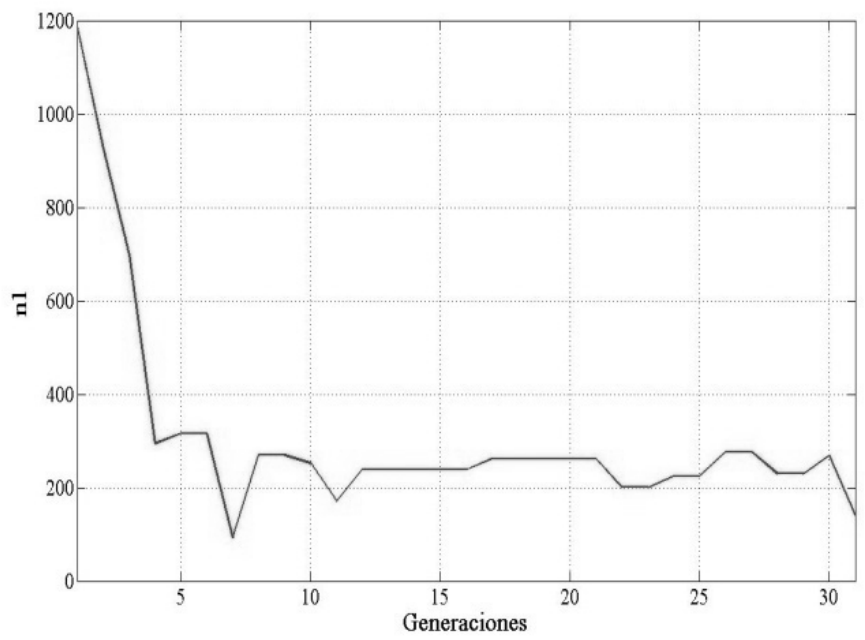

FIG. 2. EVOLUCIÓN DE LA SENSIBILIDAD DEL VALOR PROPIO $\Delta \omega$

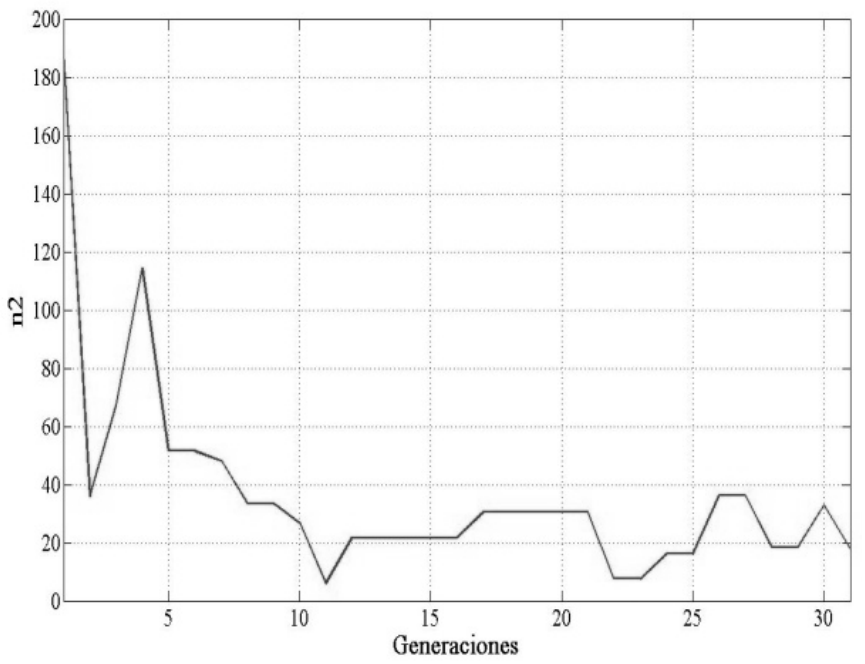

FIG. 3. EVOLUCIÓN DE LA SENSIBILIDAD DEL VALOR PROPIO $\Delta \mathrm{i}_{\mathrm{d}}$ 


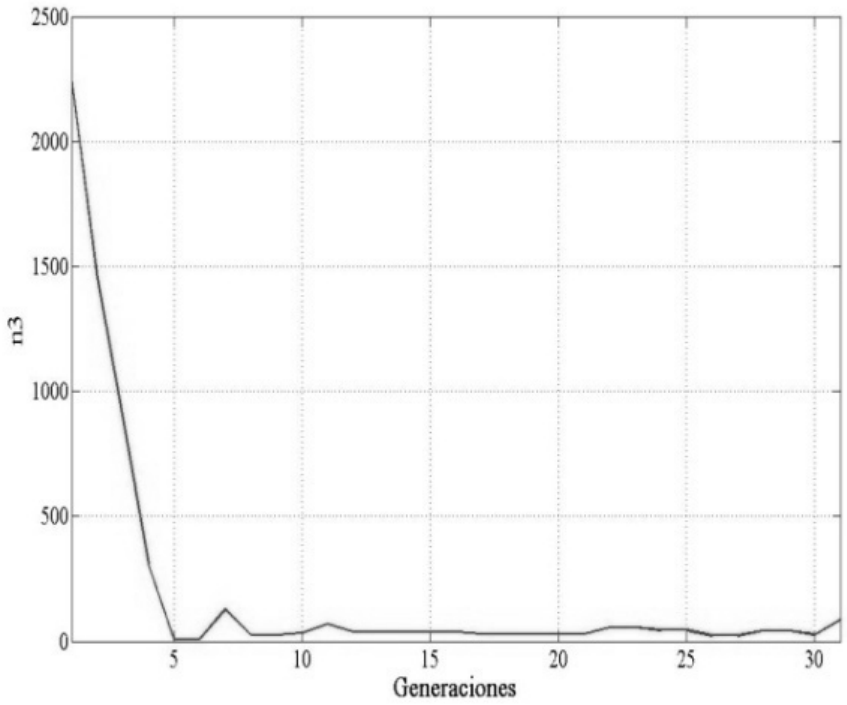

FIG. 4. EVOLUCIÓN DE LA SENSIBILIDAD DEL VALOR PROPIO $\Delta \mathrm{i}_{\mathrm{q}}$

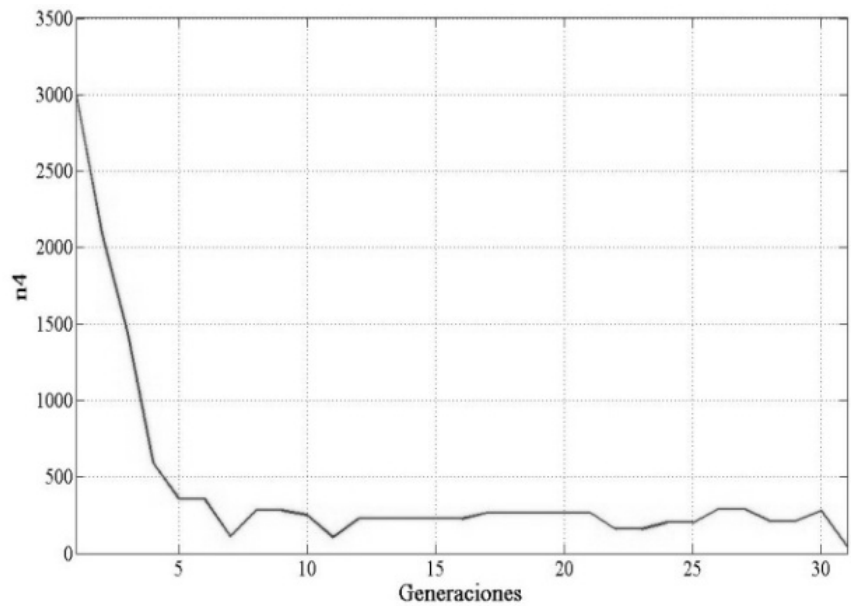

FIg. 5. EVOLUCIÓN DE LA SENSIBILIDAD DEL VALOR PROPIO $\Delta \phi_{\mathrm{d}}$ 


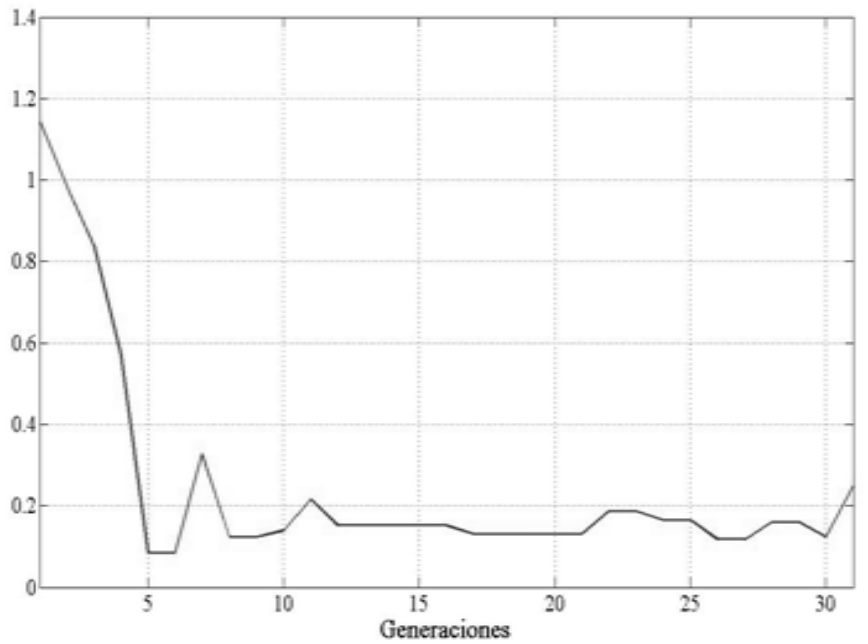

FIG. 6. EVOLUCIÓN DE LA FUNCIÓN DE DESEMPEÑO DEL CONTROL ROBUSTO

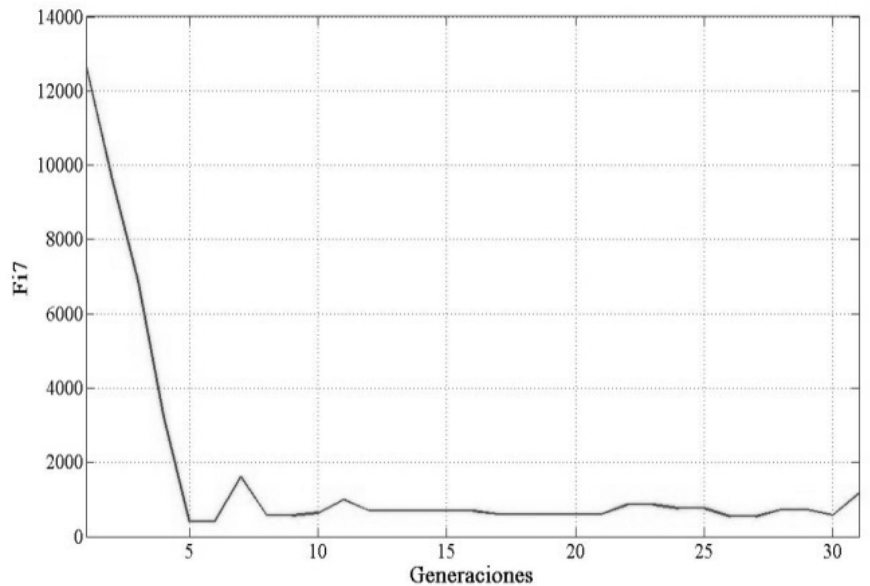

FIG. 7. EVOLUCIÓN EN LA GANANCIA K 
Los parámetros del mejor regulador encontrado dentro del algoritmo se observan en (37) y los valores propios óptimos en (38).

$$
\begin{aligned}
& \mathrm{K}_{\mathrm{op}}=\left[\begin{array}{llll}
0,4630 & 0,2517 & 1,1140 & 0,9545 \\
0,2480 & 0,9957 & 0,6916 & 0,4969
\end{array}\right] \\
& \lambda_{\text {op }}=[-485,1-1328,4-3181,4-3483,6]
\end{aligned}
$$

Los índices de desempeño que hace parte del mejor regulador son $\phi_{\mathrm{i}}=170,9753, \phi_{2}=5,9984, \phi_{3}=69,1032, \phi_{4}=105,1939, \phi_{5}=$ 0,2163 y $1,0053 \times 10^{3}$.

$\mathrm{Al}$ aplicar el regulador al sistema como se muestra en la Fig. 1; que representa un regreso al punto de operación seleccionado desde una condición inicial. Se observan en la Fig. 8 cinco pruebas para la velocidad angular con ganancias obtenidas por el AG mono objetivo y la Fig. 9 presenta el caso del AG múltiple objetivo, donde se tiene la respuesta para cuatro matrices de ganancia y la ganancia óptima; también se presenta la prueba de robustez para el sistema con perturbación de ruido blanco y se observa la regulación en la velocidad. Las Fig. 10 y 11 presentan la forma en que las ganancias de la población del AG llevan la intensidad de eje directo y cuadratura al punto de operación; de allí se visualiza la ganancia óptima (Kop) regulando sin sobre pasos las variables de estado con optimización de múltiple objetivo.

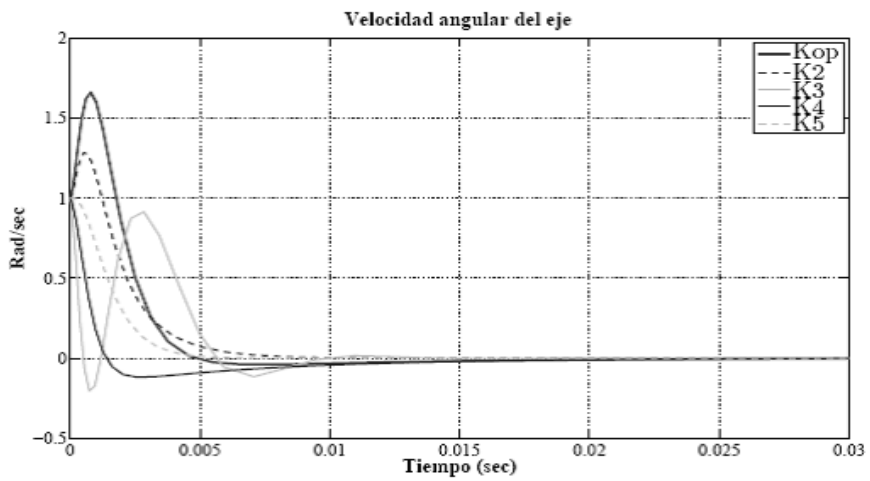

Fig. 8. Regulación de LA VARIAble de estado $\Delta \omega$, caso mono objetivo 

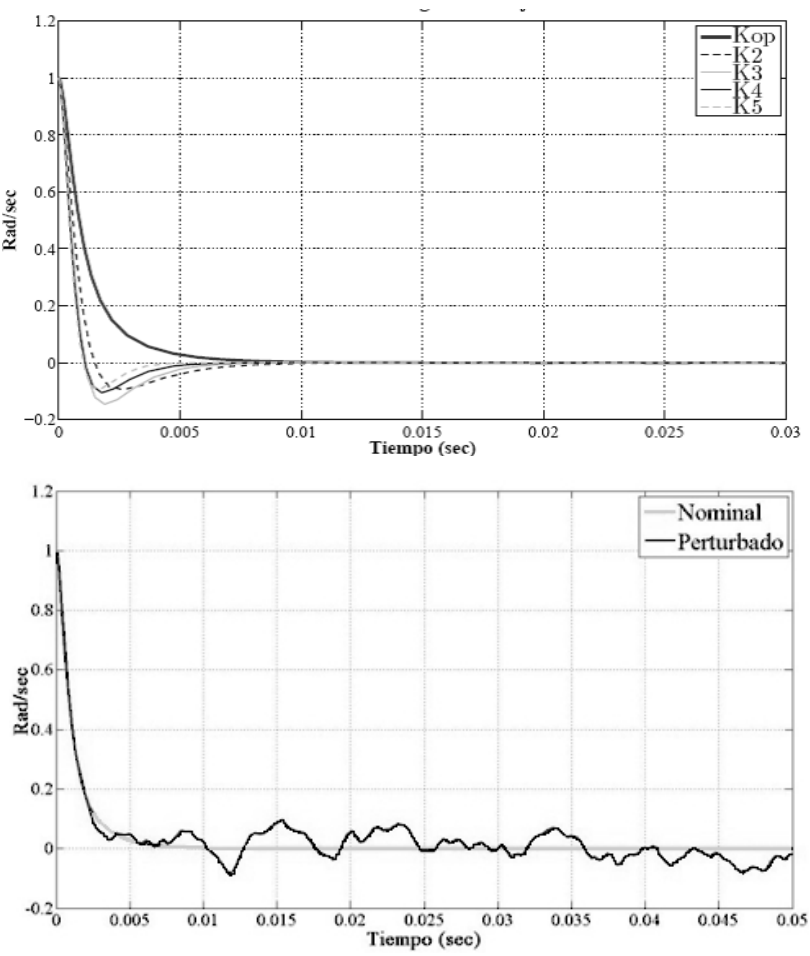

Fig. 9. Regulación de LA VARIABLE de ESTAdo $\Delta \omega$, CASO Múltiple objetivo y PRUeba DE ROBUSTEZ

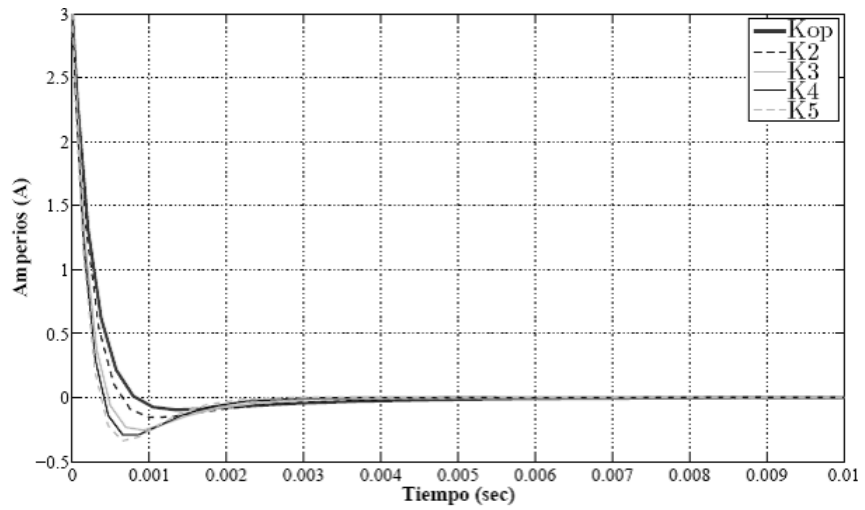

Fig. 10. Regulación de LA VARIAble de EStAdo $\Delta_{\text {id }}$, CASO MÚltiple objetivo 


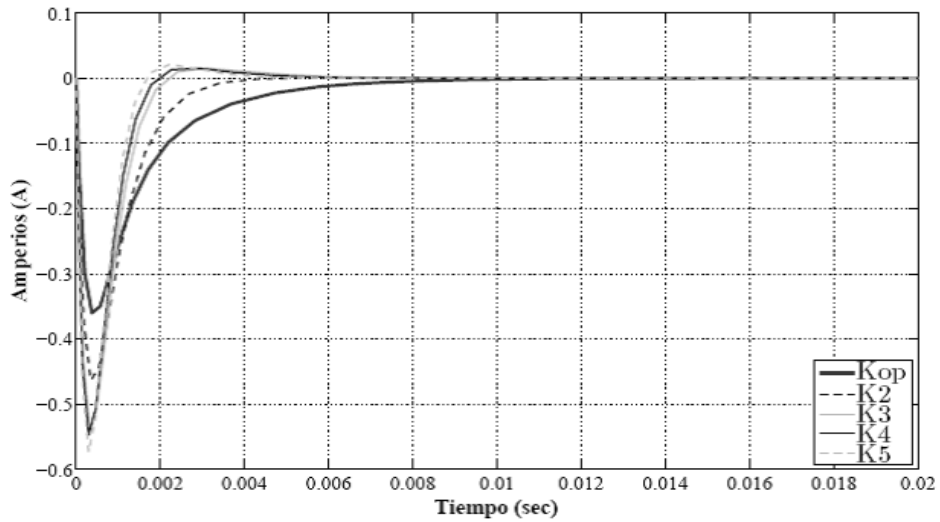

Fig. 11. Regulación de LA VARIABLE de estado $\Delta_{\text {iq }}$, CASO Múltiple objetivo

El comportamiento del sistema en lazo cerrado obtenido en la última generación de AG de múltiple objetivo y con cinco individuos de la población incluyendo el Kop infiriendo en la variable de estado $\Delta \phi_{d}$ se muestra en la Fig. 12.

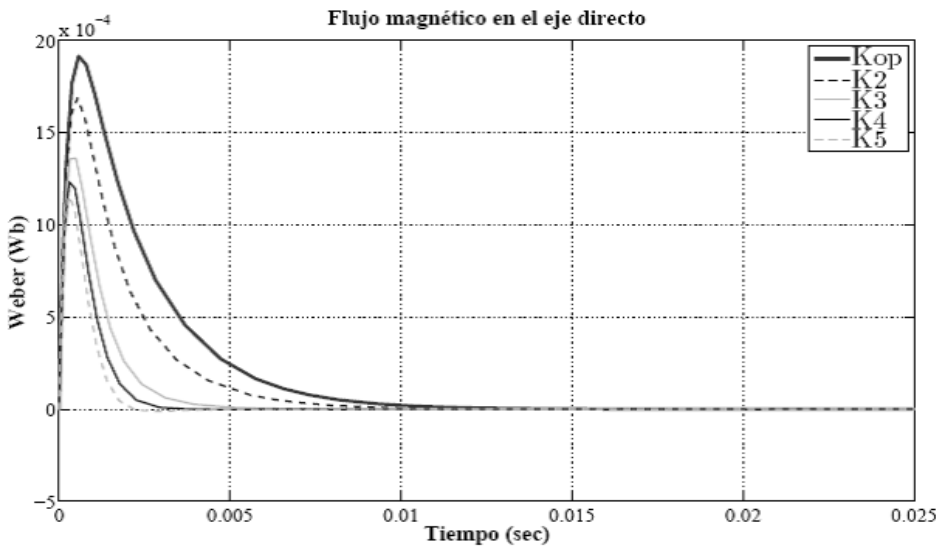

Fig. 12. Regulación de LA VARIABLE DE ESTADo $\Delta \phi_{d}$ 
El controlador hallado a parte de garantizar la regulación y una sensibilidad en cada una de las variables de estado, también garantiza unas propiedades robustas como es el comportamiento nominal (NP) y la estabilidad robusta (RP). Las Fig. 13 y 14 muestran el comportamiento nominal del sistema adecuado debido a que al aumentar la frecuencia los valores singulares en cada una de las variables de estado tiende a cero y la estabilidad robusta disminuye a igual ritmo.

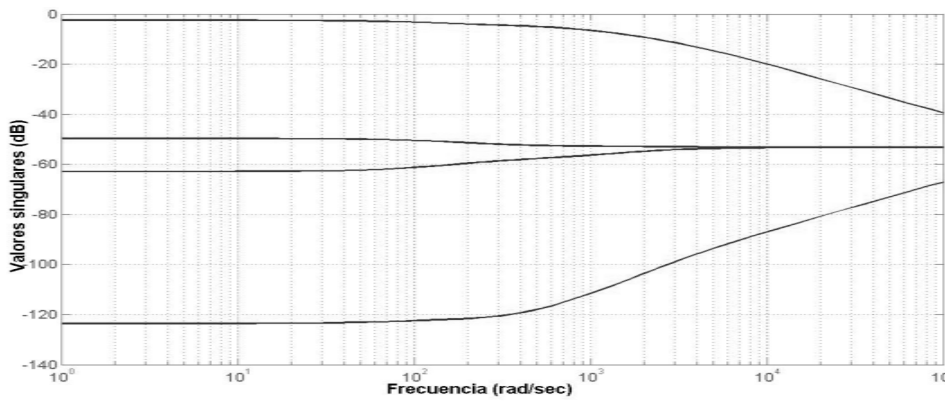

Fig. 13. VALORES SINGULARES DEL COMPORTAMIENTO NOMINAL PARA CADA VARIABLE DE ESTADO

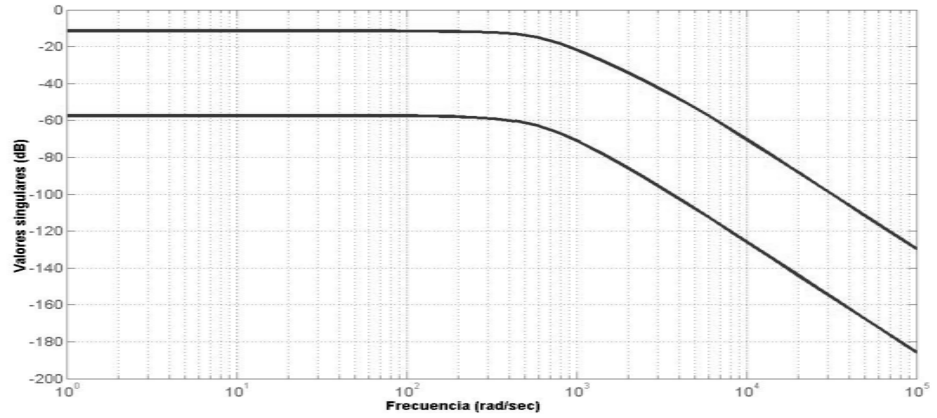

Fig. 14. VALORES SINGULARES EN LA ESTABILIDAD ROBUSTA PARA CADA VARIABLE DE ESTADO 
La Tabla 1 muestra la comparación entre las técnicas de control $\mathrm{H}_{\infty}$, el AG simple y el AG de múltiple objetivo; de allí se observa como la ponderación y normalización de las funciones objetivo para la asignación de estructuras propias caso múltiple objetivo (EP-AG-MO) es menor que el caso mono objetivo (UO) y el índice de desempeño sensibilidad para el control $\mathrm{H}_{\infty}$.

Tabla 1. Comparación de LAS tÉCNICAS de CONTROL

\begin{tabular}{lc}
\hline Controlador & Índice de desempeño \\
\hline $\mathrm{H}_{\infty}$ & 0.6484 \\
EP-AG-UO & 0.7469 \\
EP-AG-MO & 0.2443 \\
\hline
\end{tabular}

\section{Conclusiones}

Se usó un controlador lineal fijo para un motor de inducción modelado en el sistema de coordenadas de flujo del rotor cuyas entradas son los voltajes en el eje directo y en el eje de cuadratura. La sintonización del controlador presenta como objetivos garantizar una estabilidad nominal, propiedades robustas como es el comportamiento nominal y la estabilidad robusta, minimizar la sensibilidad en los polos del sistema frente a perturbaciones de forma individual y la ganancia en el controlador. Logrando así que el objetivo del controlador sea alcanzado por la implementación de un sistema de optimización heurístico de múltiple objetivo.

En este trabajo el uso de funciones de desempeño cumple el objetivo de mejorar las propiedades de robustez en un rango de frecuencias, la disminución del tiempo de respuesta de cada variable de estado y reducción del valor de la ganancia del regulador para que de esta forma las tensiones de entrada no lleguen a niveles superiores a los de operación correcta.

El uso de la asignación de estructuras propias con optimización de múltiple objetivo en este trabajo genera que el polinomio característico del sistema se mantenga dentro de los límites dados 
por el diseñador y se halle un controlador con minimización de la energía de entrada.

\section{Agradecimientos}

Este trabajo está en el marco del proyecto de investigación control de turbinas eólicas realizado en convenio e inscrito en los centros de investigación de la Universidad Tecnológica de Pereira y el Instituto Tecnológico Metropolitano, Institución Universitaria, Adscrita a la Alcaldía de Medellín.

\section{ReFERENCIAS}

Bose K., (2002); Modern Power Electronics and AC Drives, Prentice Hall, United States.

Chiasson, J., (1998); A new approach to dynamic feedback linearization control of an induction motor, IEEE Transactions on Automatic Control, 43, 391-397.

Chiasson, J., (2005); Modeling and High Performance Control of Electric Machines. IEEE Press Series on power Energy, Wiley-Interscience, New Yersey.

Duval, C., Clerc, G., \& Le Gorrec, Y., (2006); Induction machine control using eigenstructure assignment, Control Engineering Practice, 14, 29-43.

Leonhard, W., (2001); Control of electrical drives. Srpinger, New Yersey, third edition.

Liu, G.P., Patton, R.J., (1997); Eigenstructure Assignment for control System Design, John Wiley and Sons.

Liu, G.P., Patton, R.J., (1998a); Robust control design of descriptor system using eigenstructure assignment, UKACC International Conference on CONTROL IEEE, 451-456.

Liu, G.P., Patton, R.J., (1998b); Low sensitive and robust control design via output feedback eigenstructure assignment, UKACC International Conference on Control, 457-462.

Mohanti, K.B., De, N.K., (2006); Linearizing control of an induction motor, Industrial Technology, ITIT 2006, IEEE International conference on industrial technology, 45-50. 
Rodríguez, F., López, M.J., (1996); Control Adaptativo y Robusto, Universidad de Sevilla, España.

Sánchez, S., Giraldo, E., Giraldo, D., (2008); Speed Control of Induction Motor Using Robust Control with LSPD, IEEE Electronics, Robotics and Automotive Mechanics Conference, 350-353.

Ustun, S.V., Dermitas, M., (2009); Modeling and control of V/f controlled induction motor using genetic-ANFIS algotihm, Energy Converison and Management, 50, 786-791.

Whidborne, J.F., Liu, G.P., Patton, R.J., (2002); Multiobjetive design using various control techniques, IEEE international Symposium on computer Aided Control System Design Proceedings, 1-6.

Whidborne, J.F., Liu, G.P., Yang J.B., (2003); Multiobjective Optimization and Control, Research studies press ltd, Great Britain. 